\title{
Exact ground-state properties of a one-dimensional Coulomb gas
}

\author{
G. E. Astrakharchik ${ }^{1}$ and M. D. Girardeau ${ }^{2}$ \\ ${ }^{1}$ Departament de Fisica i Enginyeria Nuclear, Campus Nord B4, Universitat Politècnica de Catalunya, E-08034 Barcelona, Spain \\ ${ }^{2}$ College of Optical Sciences, University of Arizona, Tucson, Arizona 85721, USA
}

(Received 14 February 2011; published 15 April 2011)

\begin{abstract}
The ground-state properties of a single-component one-dimensional Coulomb gas are investigated. We use Bose-Fermi mapping for the ground-state wave function which permits solution of the Fermi sign problem in the following respects: (i) the nodal surface is known, permitting exact calculations; and (ii) evaluation of determinants is avoided, reducing the numerical complexity to that of a bosonic system and, thus, allowing simulation of a large number of fermions. Due to the mapping, the energy and local properties in one-dimensional Coulomb systems are exactly the same for Bose-Einstein and Fermi-Dirac statistics. The exact ground-state energy is calculated in homogeneous and trapped geometries using the diffusion Monte Carlo method. We show that in the low-density Wigner crystal limit an elementary low-lying excitation is a plasmon, which is to be contrasted with the high-density ideal Fermi gas/Tonks-Girardeau limit, where low-lying excitations are phonons. Exact density profiles are compared to the ones calculated within the local density approximation, which predicts a change from a semicircular to an inverted parabolic shape of the density profile as the value of the charge is increased.
\end{abstract}

DOI: 10.1103/PhysRevB.83.153303

PACS number(s): 71.10.Pm, 71.10.Hf, 73.21.Hb

The recent progress in nanoscale technology has made it possible to realize clean one-dimensional quantum gases, such as ultracold atoms confined in elongated traps ${ }^{1}$ and electrons in single-well carbon nanotubes ${ }^{2}$ and in semiconductor quantum wires. ${ }^{3}$ A peculiarity of a one-dimensional world is that such systems can be explained not by the conventional Landau theory of normal Fermi liquids but, rather, by an effective low-energy Luttinger liquid description, ${ }^{4}$ generalizable to long-range Coulomb interactions. ${ }^{5}$ A number of approaches of increasing accuracy (random-phase approximation, SingwiTosi-Land-Sjölander scheme, density functional theory) have been devised to study energetic and structural properties. ${ }^{6}$ The most precise calculations of energy have been obtained by the Monte Carlo technique as in Ref. 7, where a quasi-onedimensional geometry with a finite width of the transverse confinement is studied. The purpose of the present work is to carry out exact calculations in a strictly one-dimensional geometry.

In this paper we use the Bose-Fermi mapping to find the ground-state energy of a one-dimensional single-component Coulomb system exactly within statistical precision. The mapping applies to both homogeneous and trapped geometries.

We consider a single-component system of $N$ particles (bosons or fermions) of charge $e$ and mass $m$ in a onedimensional box of length $L$. Periodic boundary conditions are applied and the Coulomb potential is truncated for interparticle distances larger than $L / 2$. The Hamiltonian reads

$$
\hat{H}=-\frac{\hbar^{2}}{2 m} \sum_{i=1}^{N} \frac{\partial^{2}}{\partial z_{i}^{2}}+\sum_{i<j}^{N} \frac{e^{2}}{\left|z_{i}-z_{j}\right|} .
$$

Natural length scales in a homogeneous system are defined by atomic units, that is, the Bohr radius $a_{0}=\hbar^{2} / m e^{2}$ for length and the Rydberg number $R y=e^{2} / 2 a_{0}$ for energy. The system properties are governed by a single parameter, fixed by the ratio of Bohr $a_{0}$ radius and Wigner-Seitz $r_{s}=1 / 2 n$ radius or, equivalently, by the dimensionless density $n a_{0}$ with $n=N / L$.

Hamiltonian (1) describes a system with the pure Coulomb interaction $V_{\text {int }}(r)=e^{2} / r$ between charges not screened while the movement of charges is confined to a quantum wire, so that excitation of the levels in the transverse direction is not possible and the system shows quasi-one-dimensional behavior. For a harmonic transverse confinement this implies that the energy associated with the Hamiltonian, Eq. (1), is low compared to $N \hbar^{2} / m b^{2}$, where $b$ is the transverse oscillator length. The peculiarity of the Coulomb interaction in a one-dimensional geometry is that its Fourier transform cannot be calculated due to a divergence at short distances. Commonly, this ultraviolet divergence is cured by considering the ground state of the transverse harmonic oscillator. This effectively introduces a cutoff to the interaction potential and removes the divergence as $V_{\text {int }}^{\text {eff }}(0)=\sqrt{\pi} e^{2} / b$. Here, instead, we note that the unbound potential in Eq. (1) will not produce any divergence in the energy, while the pair distribution function vanishes when two particles approach each other. This permits us to successfully apply the one-dimensional Bose-Fermi mapping ${ }^{8}$ and relate the many-body ground-state wave functions of fermions $\psi_{F}\left(z_{1}, \ldots, z_{N}\right)$ and bosons $\psi_{B}\left(z_{1}, \ldots, z_{N}\right)$ as

$$
\psi_{B}\left(z_{1}, \ldots, z_{N}\right)=\left|\psi_{F}\left(z_{1}, \ldots, z_{N}\right)\right| .
$$

In the case of bosons the divergent $1 / r$ interaction mimics the Pauli exclusion principle, which, for fermions, prohibits any two fermions from overlapping.

We resort to the diffusion Monte Carlo (DMC) technique for finding the ground-state properties of the system. The guiding wave function is chosen in bosonic Bijl-Jastrow form, adding antisymmetrization in the case of fermions:

$$
\psi_{F}\left(z_{1}, \ldots, z_{N}\right)=\prod_{i<j}^{N} f_{2}\left(\left|z_{i}-z_{j}\right|\right) \operatorname{sgn}\left(z_{i}-z_{j}\right) .
$$

It is well known that simulations of fermionic systems suffer from the "sign problem." In terms of the DMC algorithm this means that a "fixed-node" approximation should be used; that is, the fermionic ground-state wave function is expressed as some non-negative function multiplied by a function that has a certain nodal surface. The DMC method finds the best energy 
for a given choice of nodal surface, providing an upper bound for the ground-state energy. The exact ground-state energy is reached only if the nodal surface is exact. Importantly, the nodal surface of Eq. (3) is exact. This means that the mapping, Eq. (3), permits us to find the exact energy of the fermionic system in DMC calculation. Furthermore, the same mapping allows avoidance of evaluation of determinants and reduces the complexity of the calculation from $N^{3}$ to $N^{2}$, making a larger number of particles accessible.

We choose the two-body correlation term $f_{2}(z)$ as

$$
f_{2}(z)= \begin{cases}C_{1} \sqrt{z} I_{1}(2 \sqrt{z}), & |z|<R_{\mathrm{par}}, \\ \sin ^{C_{2}}(\pi|z| / L), & |z|>R_{\mathrm{par}}\end{cases}
$$

where coefficients $C_{1}$ and $C_{2}$ are fixed by the continuity conditions for $f_{2}(z)$ and $f_{2}^{\prime}(z)$ at the matching distance $R_{\mathrm{par}}$. The short-range part of $f_{2}(z)$ corresponds to the zero-energy solution of the two-body scattering problem. This avoids divergences in the energy as two particles come close to each other. The long-range part of $f_{2}(z)$ is taken from a hydrodynamic theory. ${ }^{9}$ The parameter $R_{\text {par }}$ is optimized by minimization of a variational energy. DMC density profiles and $S(k)$ are calculated using the technique of pure estimators. ${ }^{16}$

The typical kinetic energy per particle scales quadratically with the density, opposite to the linear density dependence in the Coulomb interaction energy. At high densities the kinetic energy becomes dominant and can be approximated by the energy of an ideal Fermi gas (IFG):

$$
\frac{E^{\mathrm{IFG} / \mathrm{TG}}}{N}=\frac{\pi^{2} \hbar^{2} n^{2}}{6 m},
$$

where TG stands for Tonks-Girardeau gas. In the opposite regime of low density, the energy can be obtained by summation of the Coulomb potential energy under the assumption that all particles are localized at lattice sites of a Wigner crystal. The leading contribution to the energy (see, e.g., Ref. 10),

$$
\frac{E^{\text {Wigner }}}{N}=e^{2} n \ln N,
$$

for a fixed number of particles is linear in the density. Instead, for a fixed density the energy per particle and the chemical potential diverge logarithmically as the number of particles increases. This is a consequence of the long-range nature of the Coulomb interaction potential. In a three-dimensional system a similar divergence can be cured by the "jellium" model, that is, by restoring charge neutrality by adding a uniform charge of the opposite sign. In a one-dimensional system the introduction of a uniform opposite charge would lead to a logarithmic divergence in the short-range part of the Coulomb interaction potential.

The energy of a homogeneous system is shown in Fig. 1. At low densities the energetic properties are precisely captured by the Wigner crystal description. At the same time, no true crystal is formed, as quantum fluctuations destroy the diagonal longrange order in a one-dimensional geometry. ${ }^{11}$ The high-density regime is well described by an IFG or a TG gas, depending on the particle statistics. There is a smooth crossover between the Wigner crystal and the IFG/TG gas regimes.

Low-lying excitations in gases with short-range potentials are phonons with a linear dispersion relation $\omega_{k}=|k| c$, where $c$ is the speed of sound. This is the case of $\delta$-interacting

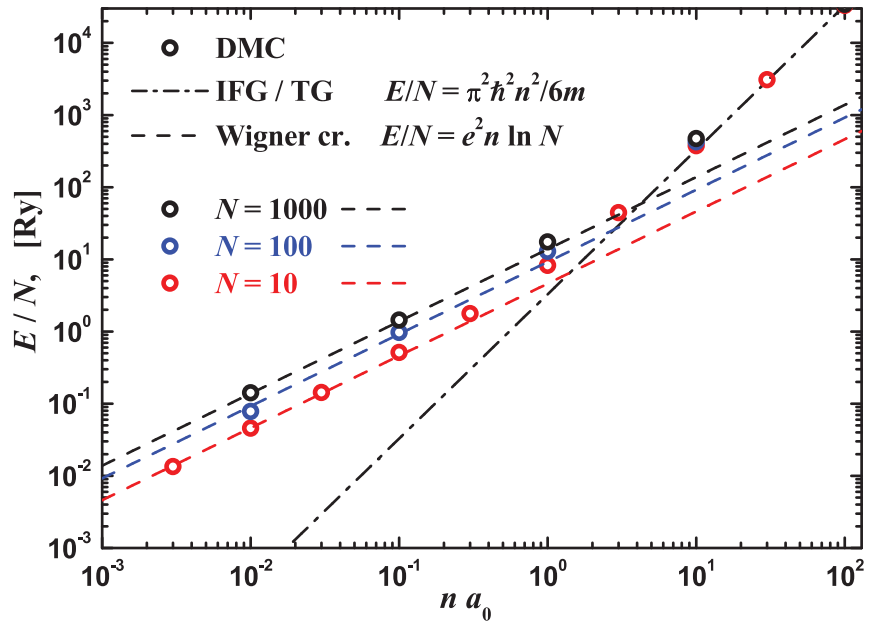

FIG. 1. (Color online) Energy per particle as a function of the dimensionless density for $N=1000,100$, and 10 (top to bottom) particles. Circles, Monte Carlo results; dash-dotted line, IFG/TG energy, Eq. (5); dashed lines, energy of a Wigner crystal, Eq. (6).

Lieb-Liniger, ${ }^{12}$ Calogero-Sutherland, ${ }^{13}$ and $\mathrm{TG}^{8}$ gases, in which the long-range properties of the correlation functions are described by the Luttinger liquid theory. ${ }^{4}$ The long-range nature of the Coulomb interaction potential leads to different low-lying excitations which instead are plasmons. Their dispersion relation can be found from the classical equations of motions in a Wigner crystal.,14 The low-momentum dependence is nonanalytic and contains logarithmic terms:

$$
\omega_{k}^{2}=\frac{2 e^{2} n}{m}\left|\ln \frac{k}{n}\right| k^{2}+\frac{3 e^{2} n}{m} k^{2}+O\left(k^{4}\right) .
$$

When the excitation spectrum is exhausted by one branch of excitations, the excitation spectrum is related to the static structure factor $S_{k}=\left\langle\rho_{k}^{\dagger} \rho_{k}\right\rangle / N$ by the Feynman formula $S_{k}=$ $\hbar^{2} k^{2} /\left(2 m \omega_{k}\right)$. We perform a DMC calculation of the static structure factor and present the results in Fig. 2. We observe that the low-energy charge excitations are well described by the plasmonic dispersion relation, Eq. (7). We note that although the logarithmic term in Eq. (7) mathematically is the leading one, it alone is not enough to describe the excitation spectrum accurately and the use of the subsequent term is very important. In the opposite regime of high density the dispersion relation is similar to that of an IFG, and the low-lying excitations are phonons.

The long-range nature of the Coulomb potential causes no divergences in finite-size trapped systems. We consider the case of a harmonic oscillator and add the confinement term $V_{\text {trap }}(z)=m \omega^{2} z^{2} / 2$ to Hamiltonian (1). It is convenient to use oscillator units of $\hbar \omega$ and oscillator length $a_{\mathrm{osc}}=\sqrt{\hbar / m \omega}$ for energy and distances, respectively. In dimensionless units the Hamiltonian reads

$$
\hat{H}_{\mathrm{dim}}^{\mathrm{trap}}=-\frac{1}{2} \sum_{i=1}^{N} \frac{\partial^{2}}{\partial z_{i}^{2}}+\frac{1}{2} \sum_{i=1}^{N} z_{i}^{2}+\sum_{i<j}^{N} \frac{q^{2}}{\left|z_{i}-z_{j}\right|},
$$

and the system is characterized by the number of particles $N$ and the dimensionless charge $q=\sqrt{a_{\mathrm{osc}} / a_{0}}$. 


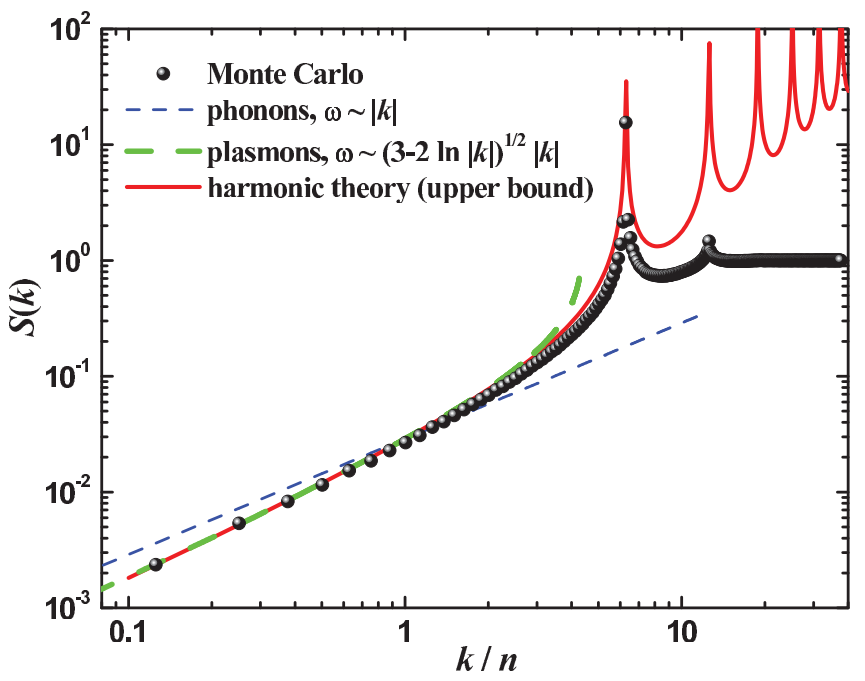

FIG. 2. (Color online) Static structure factor for $N=100$ particles in the Wigner crystal regime $\left(n a_{0}=0.01\right)$. Circles, DMC results. Lines: Feynman relation $S_{k}=\hbar^{2} k^{2} /\left(2 m \omega_{k}\right)$ for different dispersion relations-phonons and plasmons, Eq. (7); harmonic theory $[\hbar \omega(k) / R y]^{2}=4 \zeta(3)-2 L i_{3}\left(e^{i k / n}\right)-2 L i_{3}\left(e^{-i k / n}\right) .{ }^{14}$

For a small number of particles the density profile has a typical shell structure of a mesoscopic system as shown in Fig. 3. When the shell structure can be neglected, the system properties are well described within the local density approximation (LDA). ${ }^{15}$ The chemical potential $\mu$ of the trapped system is then approximated by the sum of the external potential $V_{\text {trap }}(z)$ and the chemical potential $\mu_{\text {hom }}(n)$ of the homogeneous system, with the local density $n(z)$. The value of the chemical potential is fixed by the normalization condition $N=\int n(z) d z$.

In the limit of low charge $q \rightarrow 0$, the chemical potential of a homogeneous system as in Eq. (5) can be approximated by a kinetic energy of an IFG $\mu_{\text {hom }}=\pi^{2} \hbar^{2} n^{2} / 2 m$ and is

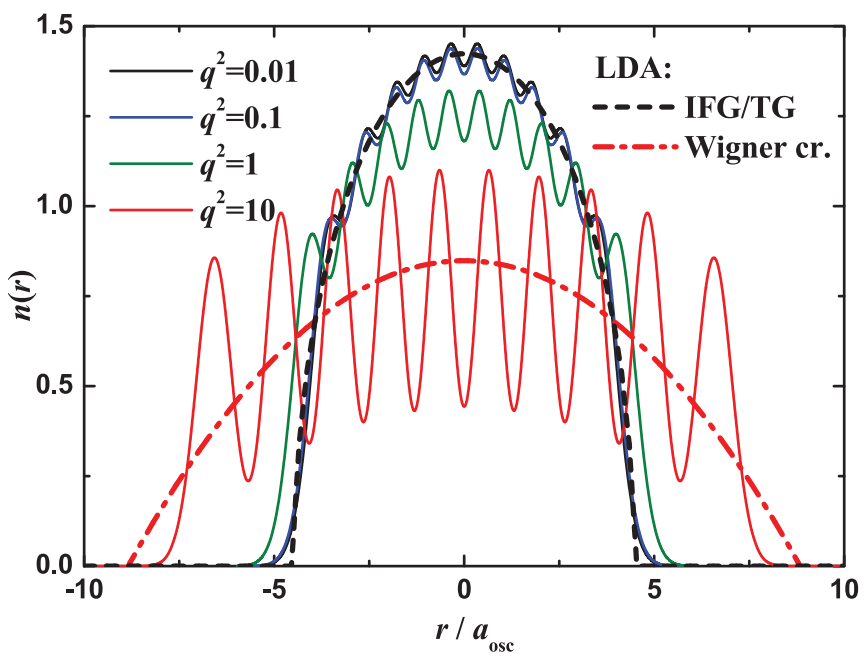

FIG. 3. (Color online) Density profile of $N=10$ trapped particles. Solid line, diffusion Monte Carlo result. Local density approximation with equation of state as in an ideal Fermi gas (dashed line) and Wigner crystal (dash-dotted line). independent of particle charges. The density profile is a semicircle of a Thomas-Fermi size $R=\sqrt{2 N} a_{\mathrm{osc}}$ :

$$
n(r)=\frac{1}{\pi} \sqrt{R^{2}-r^{2}} .
$$

The chemical potential is $\mu=N \hbar \omega$ and the energy per particle is $E / N=N \hbar \omega / 2$. Even if the LDA does not describe the shell structure, thus leaving out contributions of the order of $1 / N$, the obtained LDA result for the energy turns out to be exact for a trapped IFG.

In the limit of high charge $q \rightarrow \infty$, the chemical potential of a homogeneous system has a linear dependence on the density $\mu_{\text {hom }}=2 e^{2} n \ln N$, which is obtained by differentiating the Wigner crystal energy, Eq. (6). The density profile has an inverted parabola shape, typical for gases with short-range interaction potentials, ${ }^{15}$

$$
n(r)=\frac{1}{4 q^{2} \ln N}\left(R^{2}-r^{2}\right),
$$

with Thomas-Fermi radius $R=\left(3 N \ln N q^{2}\right)^{1 / 3}$. The chemical potential is $\mu=1 / 2\left(3 q^{2} N \ln N\right)^{2 / 3}$ and the energy per particle $E / N=3 / 10\left(3 q^{2} N \ln N\right)^{2 / 3}$.

We adapt the guiding wave function, Eq. (3), of a homogeneous system by multiplying it by one-body terms $f_{1}\left(z_{i}\right)=\exp \left(-\alpha z_{i}^{2}\right)$, with the free parameter $\alpha$ optimized by minimizing the variational energy. The two-body terms are chosen as $f_{2}(z)=\sqrt{z} I_{1}(2 \sqrt{q z})$.

Figure 4 shows the energy of $N$ charged particles in a harmonic trap. For low charges the energy per particle is well described by that of an IFG and increases linearly with the number of particles $N$. Each new particle occupies the next $l=N-1$ level of the harmonic oscillator and the energy increases by $E_{l}=(l+1 / 2) \hbar \omega$. For high charges the LDA result with the Wigner crystal equation of state applies. In this regime the energy per particle increases as $(N \ln N)^{3 / 2}$. In between the two limits there is a smooth crossover.

The density profile for a system of $N=10$ is presented in Fig. 3. For low charges the LDA predicts a semicircular shape

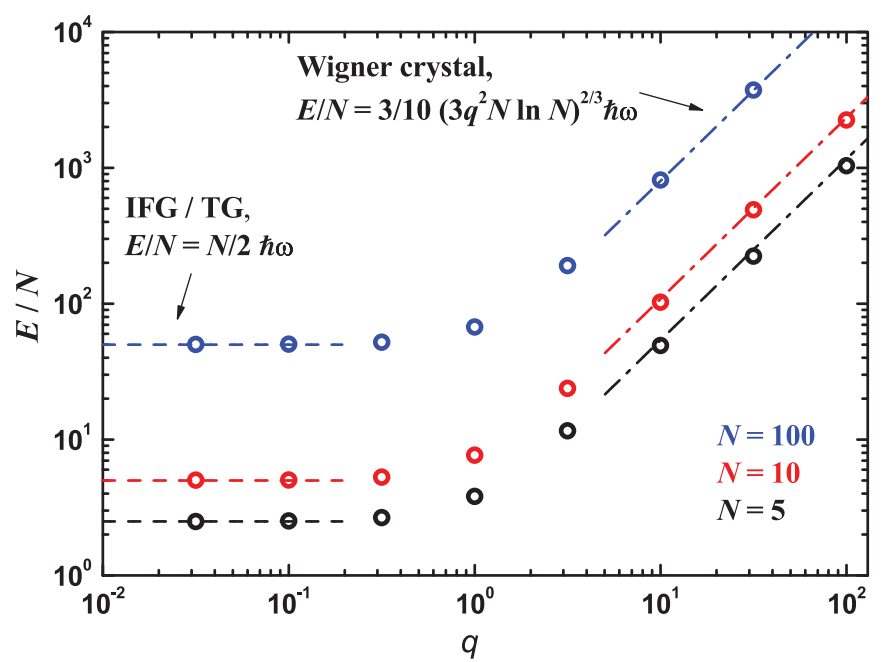

FIG. 4. (Color online) Ground-state energy of $N=100,10$, and 5 (top to bottom) charged particles in a harmonic trap. Circles, DMC results; dashed line, energy of IFG/TG gas; dash-dotted line, LDA result for Wigner crystal. 
of a radius that depends only on the number of particles and is independent of the value of the charge. For that reason different density profiles with $q^{2} \lesssim 0.1$ almost coincide. The regime of high charges is a trap analog of a Wigner crystal. The particles are highly localized and form well-defined shells, while the overall shape is an inverted parabola of a width that depends on the value of the charge. Differently from a homogeneous Wigner crystal, here the distance $a$ between lattice sites is no longer constant and depends on the distance $z$ from the center of the trap as $a(z) \approx 1 / n(z)$. The LDA description is known to be imprecise at the borders of the system, ${ }^{15}$ as it predicts cusps in the density, while the true density profile is smooth as shown in Fig. 3. Furthermore, the high-density Wigner crystal equation of state is not applicable at the edges where the density is low, which worsens the LDA description of a trapped Wigner crystal at the edges. The square of the size of the system is related to the potential energy of the trap and has a behavior similar to that of the total energy (see Fig. 4).

To conclude, we have studied the properties of a singlecomponent Coulomb $1 / r$ gas in a one-dimensional geometry. The short-range divergence of the interaction potential permits us to exploit the Fermi-Bose mapping for the ground-state wave function and to solve the Fermi sign problem in the system considered, as (i) the mapping allows exact evaluation of the ground-state properties, and (ii) the numerical complexity of a simulation in a fermionic system is the same as in a bosonic system. This permits us to carry out ab initio simulations of many-body systems with as many as 1000 fermions. Due to the mapping, the bosonic and fermionic Coulomb systems have the same energy and local properties (density profile, pair distribution functions, etc.).

We use the DMC method to do an exact calculation of the ground-state energy of a system in a box with periodic boundary conditions and in a harmonic trap. In the regime of high density a homogeneous system is well described by an IFG for fermions or a TG gas for bosons. There is a smooth crossover transition to the Wigner crystal regime as the density is lowered. We show that the low-lying excitations are plasmons as manifested in the low-momentum behavior of the static structure factor.

The energy and the density profile in trapped systems are obtained within the LDA and are compared with the exact results of the DMC calculation. In the IFG/TG gas limit the system properties are defined by the number of particles and are independent of the charge. The limit of high charge is a trapped analog of a Wigner crystal where localized shells play a role similar to that of crystal lattice sites. The amplitude of the oscillations in the density profile experiences a drastic change in shape, varying from a semicircle (IFG/TG limit) to an inverted parabola (Wigner crystal limit).

G.E.A. acknowledges support from the Spanish MEC through the Ramon y Cajal fellowship program.
${ }^{1}$ B. Paredes et al., Nature 429, 277 (2004); H. Moritz, T. Stoferle, K. Guenter, M. Kohl, and T. Esslinger, Phys. Rev. Lett. 94, 210401 (2005); E. Haller et al., Science 325, 1224 (2009).

${ }^{2}$ R. Saito, G. Dresselhaus, and M. S. Dresselhaus, Physical Properties of Carbon Nanotubes (Imperial College Press, London, 1998). ${ }^{3}$ O. M. Auslaender et al., Science 308, 88 (2005); Y. Jompol et al., ibid. 31, 597 (2009).

${ }^{4}$ F. D. M. Haldane, Phys. Rev. Lett. 47, 1840 (1981); T. Giamarchi, Quantum Physics in One Dimension (Oxford University Press, London, 2004).

${ }^{5}$ H. J. Schulz, Phys. Rev. Lett. 71, 1864 (1993).

${ }^{6}$ A. Gold and L. Calmels, Solid State Commun. 96, 101 (1995);

L. Camels and A. Gold, Europhys. Lett. 39, 539 (1997); D. Agosti, F. Pederiva, E. Lipparini, and K. Takayanagi, Phys. Rev. B 57, 14869 (1998).
${ }^{7}$ M. Casula, S. Sorella, and G. Senatore, Phys. Rev. B 74, 245427 (2006); L. Shulenburger, M. Casula, G. Senatore, and R. M. Martin, ibid. 78, 165303 (2008).

${ }^{8}$ M. Girardeau, J. Math. Phys. (NY) 1, 516 (1960).

${ }^{9}$ L. Reatto and G. V. Chester, Phys. Rev. 155, 88 (1967).

${ }^{10}$ D. H. E. Dubin, Phys. Rev. E 55, 4017 (1997).

${ }^{11}$ N. D. Mermin, Phys. Rev. 176, 250 (1968).

${ }^{12}$ E. H. Lieb and W. Liniger, Phys. Rev. 130, 1605 (1963).

${ }^{13}$ F. Calogero, J. Math. Phys. 10, 2191 (1969); B. Sutherland, ibid. 12, 246 (1971).

${ }^{14}$ S. Akhanjee, e-print arXiv:0704.2088v3; S. Fishman, G. De Chiara, T. Calarco, and G. Morigi, Phys. Rev. B 77, 064111 (2008).

${ }^{15}$ F. Dalfovo, S. Giorgini, L. P. Pitaevskii, and S. Stringari, Rev. Mod. Phys. 71, 463 (1999).

${ }^{16}$ J. Casulleras and J. Boronat, Phys. Rev. B 52, 3654 (1995). 